\title{
Sound Insulation Properties of Composite Laminated Structures
}

\author{
Hanwen Zhao \\ Laboratoire de Mécanique Physique (LMP), \\ École Centrale de Pékin, Beihang University \\ hwzhao@buaa.edu.cn \\ Beijing, China
}

\author{
Jinliang $X u$ \\ Beijing Polymer Matrix Composites \\ Technology Laboratory, Beihang University \\ 14241036@buaa.edu.cn \\ Beijing, China
}

\author{
Xingrong Huang* \\ Laboratoire de Mécanique Physique (LMP), \\ École Centrale de Pékin, Beihang University \\ huangxingrong@buaa.edu.cn \\ Beijing, China \\ Le Fang \\ Laboratoire de Mécanique Physique (LMP), \\ École Centrale de Pékin, Beihang University \\ le.fang@buaa.edu.cn \\ Beijing, China
}

\begin{abstract}
The research principally consists of the qualitative and quantitative analysis of sound insulation performance of laminate structure. Composite materials have been widely used in aerospace, marine, transportation and other engineering industries due to their light weight, good mechanical properties and high temperature and corrosion resistance. On the Boeing 787 Dreamliner, more than half mass is taken by composite materials which are distributed over the wings, the empennage and the engine nacelles. As the extensive usage of composite laminated plate in the aeronautic domain, people began to focus on their acoustic performance. The mass law was proposed in the end of $19^{\text {th }}$ century (Rayleigh, 1878). It has been proved that the sound insulation is linearly dependent on the density and excitation frequency. The mass law has been widely applied and validated in different circumstances over the past century. In the middle of $20^{\text {th }}$ century, the occurrence of the theory of coincidence effect (Cremer, 1942) gave a theoretical explanation of the reduction of the acoustic performance in the middle and high band. These theories have formed the very base of modern acoustics. With the advent of computers, the study of the acoustic properties of materials has entered the era of simulation. The simulation-based studies principally use finite element analysis and statistical energy analysis. For example, the study of point-support plate structure (Shorter et al., 2005), the study of inlaid finite laminated plate ( $\mathrm{Li}$ and Zhao, 2001) and the study of sound insulation performance of glass fibre reinforced resin-based materials with steel balls inside (Yan and Huang, 2016). Many studies have been carried out on composite laminates materials, while rare conclusion or law of laminate manner constated from researches. At present,
\end{abstract}

research on mechanical properties of composite materials is becoming more and more mature, while further studies on the mechanism of sound insulation acoustic properties need to be investigated, to get a better acoustic performance of composite laminated materials.

In this paper, we firstly use the impedance analysis method to analyse the sound insulation properties. The acoustic impedance indicates the speed limit capability of a certain part of the sonic wave propagation process. The sound insulation performance can be calculated by solving the acoustic impedance. The main idea of this method is to solve the acoustic impedance of each layer of the sound insulation structure through the vibration equation, and then determine the total acoustic impedance, and then calculate the sound insulation. The impedance analysis refers to a primary preestimate of the acoustic performances. Then, the numerical experiments have been carried out to study the dependency between different laminating manners and sound insulation capabilities. The carbon fibre epoxy resin is chosen as the typical material of laminate. Then we have constructed a plate model whose dimension is $1 \mathrm{~m}^{2}$ with $1 \mathrm{~m}$ width and $1 \mathrm{~m}$ length. The total mass of the laminated plate is defined as constraint condition and it is fixed to be $4.05 \mathrm{~kg}$ to facilitate the comparison with aluminium alloy plate with the same area and $1.5 \mathrm{~mm}$ thickness. A reverberated excitation is applied as a source of sonic wave. The combined finite element statistical energy analysis method is employed to solve the problem. In order to verify the accuracy and reliability of the numerical method, we compare the simulation results with the theoretical and experimental results using an aluminium plate as example. The results show that the accuracy of the model is quite good, the relevant error of the predicted resonant frequency is limited to $3 \%$. After the verification, the aluminium plate is 
replaced by the composite laminate. The optional laminate strategies differ in number of layers, thickness of layers, orientation of the fibre etc. However, empirically, while the total mass is fixed, the orientation of each layer influences most the acoustic performance. For this reason, we select repeatably 4 orientations of laminate from 7 alternatives $0^{\circ}, 15^{\circ}, 30^{\circ}, 45^{\circ}, 60^{\circ}, 75^{\circ}$ and $90^{\circ}$, then observe the acoustic insulation performance. 42 simulations have been conducted in total, and by observing several results, some valuable conclusions can be summarized. The coincidental frequency moves from lower band to higher band when increasing the angle between 2 successive laminates, while the local minimum of acoustic insulation stays identical. Besides, if the plate contains only 2 laminating angles $\alpha$ and $\beta$, the proportion that $\alpha$ or $\beta$ takes does not influence the acoustic performance in middle-high frequency band.

The carbon laminates are wildly used in the fuselage, wing, empennage and nacelle. We hope that this work can provide inspiration for optimizing the sound insulation performance in a specific band of these structures.

\section{INTRODUCTION}

Vibro-acoustic problems are fairly common in aircraft domain. When the noise level of the machine is low, the noise level can be decreased by the structural damping. Aircraft noise is mainly generated by two mains sources: the airflow pressure pulse generated when air flows through the aircraft body and the strong noise emitted by the engine. However, in many practical situations, it is difficult to process from the noise source due to complicated environments where aircrafts locate.

We can take measures to reduce the noise on the noise propagation path. Sound insulation is one of the most efficient methods in noise control engineering. We can use the sound insulation materials and sound insulation structures to block the propagation of sound energy, to limit the noise source to a local area, or to isolate a quiet place in a noisy environment. As the extensive usage of composite laminated plate in the aeronautic domain, people began to focus on their acoustic performance.

Researchers have many studies on the sound insulation properties of composite materials. Sastry and Munjal studied the vibration distribution of infinitely large multi-layer plates excited by plane waves (Sastry and Munjal, 1995). Beranek used the vibration equation of the laminated plate to establish the sound insulation structure model of the composite laminated plate, and the calculation results are basically consistent with the experimental results (Beranek and Mellow, 2012). Reixach measured the sound transmission loss of fiber reinforced composite laminated plate by experimental methods and compared them with the simulation results, which are basically consistent (Reixach et al., 2015). Tsai and Pawar used the conjugate gradient method to estimate and optimize the relevant parameters of composite laminated plate and gave the formula of sound transmission loss of composite laminated plate (Tsai and Pawar, 2015).

\section{THEORETICAL ASPECTS} Acoustic properties of plates
There are many physical quantities in acoustics that describe the sound level, and herein sound pressure is employed. The sound pressure (P) is a pressure increase due to the presence of sound waves. We use the impedance analysis method to solve the sound transmission loss. Acoustic impedance refers to the rate limiting capability of a medium at a location. $\mathrm{Z}_{\mathrm{m}}$ is the acoustic impedance of the plate. The formula for calculating the acoustic impedance of the plate is (Du and Zhu, 2001):

$$
Z_{m}=\frac{P_{f}-P_{b}}{u}
$$

$\mathrm{P}_{\mathrm{f}}, \mathrm{P}_{\mathrm{b}}$ respectively represent the sound pressure of the front and the back of the plate, $\mathrm{u}$ is the normal vibration velocity.

The sound transmission loss (TL) is used to measure the sound insulation performance of the sound insulation structure. TL refers to the difference in sound level between the incident sound and the transmitted sound. The formula for calculating the sound transmission loss is:

$$
T L=20 \lg \left|1+\frac{Z_{m} \cos \theta}{2 \rho c}\right|
$$

c is the speed of sound, $\rho$ is the density of air, and $\theta$ is the angle of incidence.

\section{Acoustic properties of orthotropic composite laminated plates}

An infinite sound insulation model is firstly needed for composite laminates. 'Infinity is due to the fact that when the size of one direction of the structure is much smaller than the other two directions, Kirchhoff hypothesis can be employed so that the structure can treated as a two-dimensional structure (Meng and Yan, 2017).

The laminated plates studied in this paper are made of orthotropic fiber reinforced composites. The fiber reinforced composite material is composed of the fibers and the matrix material. Fibers are continuous and parallel to each other, and each fiber has the same cross section. The specific strength and specific modulus are large in the fiber direction. Matrix material bonds the fibers to maintain the relative position between them, so that the fibers are uniformly distributed in matrix material. Orthotropic refers to the fact that the chemical or physical properties of a material have different properties in two directions perpendicular to each other. The composite plates are bonded together to form a multi-angle laminated plate, assuming that the bonding between each layer is ideal and the thickness of the bond layer is negligible.

A spatial Cartesian coordinate system is established. The $\mathrm{x}$-axis and $\mathrm{y}$-axis are in the plane of the plate, and the z-axis is along the thickness of the plate. It is assuming that the laminated plate is composed of $\mathrm{n}$ plates of the same fiber reinforced composite material. The coordinates of lower surface of each plate are $h_{k-1}$, and the coordinates of upper surface are $h_{k}$.

The local direction ' 1 ' is defined by the orientation of fiber. The local directions ' 2 ' and ' 3 ' will be automatically determined by the right-hand rule. The angle between the direction ' 1 ' of each plate and the $\mathrm{x}$-axis of the coordinate system is $\alpha$. The plane wave is incident on the laminated plate 
at an angle $\theta$, and the angle between the projection of the incident wave on the plate and the $x$-axis is $\varphi$. The wavenumber of incident wave is $\mathrm{k}$, the angular frequency is $\omega$, and the surface mass density of the plate is $\mathrm{m}$. The medium on both sides of the plate is air which has the ideal fluid medium properties.

For each orthotropic composite plate, the mechanical parameters in directions ' 1 ' and ' 2 ' are different. $E_{1}$ and $E_{2}$ are the elastic modulus in directions ' 1 ' and ' 2 '. $\mathrm{v}_{12}$ is the Poisson's ratio of the transverse strain in the direction ' 2 ' caused by the normal stress in the direction ' 1 ', and $v_{21}$ is the Poisson's ratio of the transverse strain in the direction ' 1 ' caused by the normal stress in the direction ' 2 '. $G_{12}$ is the shear modulus of the plate. The stiffness coefficients are expressed as follows (Zhang and Du, 2017):

$$
\begin{gathered}
Q_{11}=\frac{E_{1}}{1-v_{12} v_{21}} \\
Q_{12}=\frac{v_{12} E_{1}}{1-v_{12} v_{21}} \\
Q_{22}=\frac{E_{2}}{1-v_{12} v_{21}} \\
Q_{66}=G_{12} \\
\bar{Q}_{11}=Q_{11} \cos ^{4} \alpha+2\left(Q_{12}+2 Q_{66}\right) \sin ^{2} \alpha \cos ^{2} \alpha+ \\
Q_{22} \sin ^{4} \alpha \\
\bar{Q}_{12}=\left(Q_{11}+Q_{22}-4 Q_{66}\right) \sin ^{2} \alpha \cos ^{2} \alpha+Q_{12}\left(\cos ^{4} \alpha+\sin ^{4} \alpha\right) \\
\bar{Q}_{22}=Q_{11} \sin ^{4} \alpha+2\left(Q_{12}+2 Q_{66}\right) \sin ^{2} \alpha \cos ^{2} \alpha+Q_{22} \cos ^{4} \alpha \\
\bar{Q}_{66}=\left(Q_{11}+Q_{22}-2 Q_{12}-2 Q_{66}\right) \sin ^{2} \alpha \cos ^{2} \alpha+Q_{66}\left(\cos ^{4} \alpha+\sin ^{4} \alpha\right)(10)
\end{gathered}
$$

$\mathrm{Q}_{11}, \mathrm{Q}_{12}, \mathrm{Q}_{22}, \mathrm{Q}_{66}$ are positive axis stiffness coefficients, $\bar{Q}_{11}, \bar{Q}_{12}, \bar{Q}_{22}, \bar{Q}_{66}$ are off-axis stiffness coefficients. Then we can calculate the bending stiffness $D_{i j}$ (Zhang and Du, 2017):

$$
D_{i j}=\frac{1}{3} \sum_{k=1}^{n}\left(\bar{Q}_{i j}\right)_{k}\left(h_{k}{ }^{3}-h_{k-1}{ }^{3}\right)
$$

With the above conditions, only the laminating sequence and laminating angles can affect the sound insulation performance. Compared to the laminating angles, the influence of the laminating sequence on the sound insulation performance is weak. The laminating sequence can be controlled by changing the anti-symmetric oblique structure, and the laminating angle becomes the only variable. The number of plates is an even number, because when the laminating angle of a certain plate is $+\alpha$, the laminating angle of the plate at its symmetrical position is $-\alpha$

Through the vibration equation of the plate (Sheng and Wang, 2001), the vibration equation of the orthotropic composite laminated plate can be simplified with antisymmetric oblique structure. When the plate is excited by sound waves, there is a relationship between the sound pressure on both sides of the plate and the normal vibration velocity, the acoustic impedance of laminated plate resulting to be:

$$
Z_{m}=j \omega m\left(1-\frac{k^{4}}{k_{b}}\right)
$$

$\mathrm{k}_{\mathrm{b}}$ is the bending wavenumber of the vibration of the laminated plate: $k_{b}^{4}=\frac{m \omega^{2}}{\sin ^{4} \theta\left(D_{11} \cos ^{4} \varphi+2\left(D_{12}+2 D_{66}\right) \sin ^{2} \varphi \cos ^{2} \varphi+D_{22} \sin ^{4} \varphi\right)}$

The formula for calculating the sound transmission loss of laminated plate with antisymmetric oblique structure is:

$$
T L=20 \lg \left|1+\frac{j \omega m \cos \theta}{2 \rho c}\left(1-\frac{k^{4}}{k_{b}{ }^{4}}\right)\right|
$$

From the formula of sound transmission loss, it can be seen that when the projection of wavenumber of the incident wave on the plate is equal to the bending wavenumber of the vibration of the laminated plate, the sound transmission loss is zero, and a coincidence effect will occur.

When a plane wave is incident on a plate at a certain angle, if the wavelength of the incident wave is exactly equal to the transverse wave wavelength of the plate, the plane wave would excite the plate to generate transverse wave resonance, and the TL would be significantly decreased. This phenomenon is called the coincidence effect, and this frequency is called the coincidence frequency $\left(\mathrm{f}_{\mathrm{c}}\right)$ (Du and Zhu, 2001). The formula for calculating the coincidence frequency is:

$$
f_{c}=\frac{\omega k_{b}^{2}}{2 \pi k^{2}}
$$

It should be pointed out that the sound transmission loss at the time of coincidence frequency is not equal to zero due to some factors such as structural damping etc. Furthermore, it can be observed from the curve of the sound transmission loss in function of frequency that, a minimum value of sound transmission loss will appear near the coincidence frequency, which will be discussed in detail from the simulation aspects in the following paragraphs.

\section{SIMULATION RESULTS AND DISCUSSIONS}

The simulation of the laminates in this paper is based on the commercial full-band acoustic simulation software VA One. The commercial software of VA One was released by the ESI Group. It contains a variety of solving modules including finite element module, statistical energy analysis module and acoustic boundary element module. The following results were obtained by using a VA One statistical energy analysis module.

The numerical experiments based on a simple realistic experiment. The plate to be tested is sandwiched between two finite acoustic chambers. The sound chamber on one side is applied with the excitation of the reverberant sound field. A significant advantage of statistical energetic analysis is the independence of discretization of the model. The numerical experiments using this method are significantly less time consuming, and the requirements for computing resources are not as high as massive parallel computing. The plate structure in this model can be any type of plate, such as a general alloy plate, a composite laminate or a honeycomb sandwich panel. In this study, our main research object is composite laminates where a fiber reinforced composite materials is applied.

Fiber reinforced composites are usually used in the laminated structure. The local direction ' 1 ' is defined by the orientation of fiber. The local directions ' 2 ' and ' 3 ' will be 
automatically determined by the right-hand rule. The anisotropy is a typical property who clearly distinguish the composite and normal alloy. While the difference of mechanical properties between the directions 2 and 3 is relatively subtle. Meanwhile, in comparison with the direction 1 , the others perform basically same. Hence, the fiber reinforced structure can be of macroscopically transverse isotropic. For multi-layered laminated structure, we can describe the structure by listing consecutively the orientation 1 of each layer. By convention, the degree symbol is generally not written in the expression. Thus, the laminated shown as the figure can be described as [-45/45/90/0].

In industry, there are just several angles available for fabrication and technology reasons. The laminating angle can only be selected from the multiple angle of $15^{\circ}$. In addition, the properties of the fiber-type material have symmetry in forward and reverse, the alternative angles are $0^{\circ}, 15^{\circ}, 30^{\circ}$, $45^{\circ}, 60^{\circ}, 75^{\circ}$ and $90^{\circ}$. Symmetric, antisymmetric and some others conventional structures exist. Without these conventional structures, there will be an enormous amount of laminate possibilities, which will greatly increase the complexity of the numerical experiments. In this paper the conventional structures are used, and the number of layers is limited as four. A simpler laminate can represent the most elementary unit of a complex multi-layered laminate, and the advantages are evident: less cost of calculation and easier to analyze.

Acoustic insulation performance comparison of aluminium alloy plate and composite laminated plates

The difference on acoustic insulation performance between a typical composite called carbon fiber epoxy resin and the aluminum alloy is analyzed at first.

While modeling the composite plate with aluminum alloy plate, it is necessary to identifying the mass as a controlled variable. Because the mass, or density, is a variable bound directly with inertia in which we are not very interested. The inertia is an important factor for noise cancellation without doubt. However, the mechanism of acoustic insulation by increasing mass or density is the same for all kinds of structure. Which means that is not necessary to stuck in the "Mass Law". Therefore, the mass of laminate plate would be determined if the dimension of aluminum alloy plate is determined. The dimension of aluminum alloy plate is $1000 \mathrm{~mm} * 1000 \mathrm{~mm} * 1.5 \mathrm{~mm}$ and it weighs $4.05 \mathrm{~kg}$.

Two typical laminating structures are selected. They are [0/30/60] and [0/45/90]. The mass is the same as the aluminum alloy plate. The acoustic insulation curve is shown as follows.

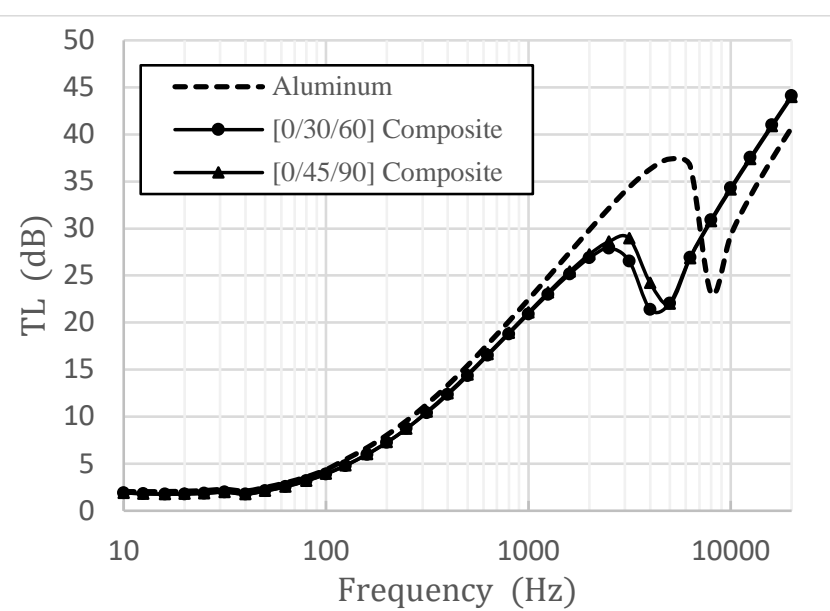

Figure 1 TL Comparison of alloy \& composite plates

In the mid-high band, the aluminum plate performs just as well as the composite. Before $1000 \mathrm{~Hz}$, the insulation performance of two materials differs by only about $1 \mathrm{~dB}$. The "Mass Law" states that the sound insulation of a wall or panel is proportional to its surface density, which is proportional to its product of density and thickness. Therefore, in this band, the mass is the most dominant factor for acoustic insulation. However, the figure shows two valleys separated by about $4000 \mathrm{~Hz}$. The aluminum plate performs obviously better than the composite. It is significant that while the local minimums of two plates are mainly the same, their local maximums differ a lot. The delay of the appearance guarantees somehow the increment of insulation curve which make the aluminum plate performs globally better than the other. Then, in the higher band, fiber epoxy resin isolates more vibration and makes its transmission loss greater than the aluminum's. As it is observed, though the TL of composite laminated plate in higher band is greater than the aluminum plate's, the rest is not. In fact, the vibration resonance is mostly characterized by relatively lower frequency. Both the source of excitation and the structure to protect are, empirically, easily excited by lowfrequency excitation. It can be therefore stated that the sound insulation performance of the carbon fiber epoxy resin laminated plate is averagely inferior than that of the aluminum plate.

\section{Studies on composite patterns in form of $[0 / \alpha]$}

Second the influence of the laminating patterns on the insulation performances is investigated.

The most determinant factors are laminate sequence, choice of angles and thickness distribution. The laminate sequence means the different orders to apply each laminate with the same thickness and the angles selected. The choice of angles means the choice from seven alternative angles with same laminating sequence. The thickness distribution means the proportion of one angle selected to another in laminate plate. These three factors play very important roles in practical applications. Besides, they are the most basic elements to describe a laminating plate. Thus, the functions to insulation performances will be discussed in detail in the following paragraphs.

Firstly, a set of experiments about the thickness distribution was realized to figure out if the thickness of one 
layer can influence the acoustic insulation property. The selected laminating angles are 0 degrees and 90 degrees. Total mass is controlled as the previous. The laminating structures are respectively $[0 / 90],[0 / 90]_{2},[0 / 90]_{3},\left[0 / 90_{2}\right]$ and $\left[0 / 90_{3}\right]$. The insulation properties of these plates show as follows.

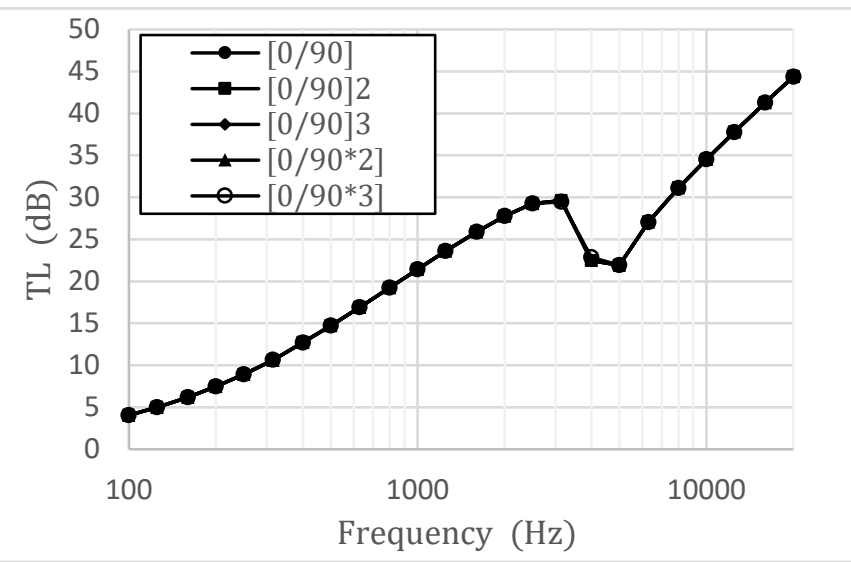

Figure 2 TL comparison of composite patterns with different proportion of lamination angles

As shown in Figure 2, while using 0 degrees and 90 degrees as two laminating angles, little difference can be observed in mid-high band and further investigations on the perpendicular structures are carried out by another set of experiments. These experiments are based on 0 degrees and 45 degrees as laminating angles. The laminating sequence $[0 / 45],[0 / 45]_{2},[0 / 45]_{3},\left[0 / 45_{2}\right]$ and $\left[0 / 45_{3}\right]$ are chosen analogically and the same numerical experiments are repeated several times. The results show that the proportion that these two selected angles do not influence the insulation property. Furthermore, if the angles between every two consecutive layers are identical, the acoustic insulation property of midhigh frequency band will be basically same.

\section{Studies on composite patterns in form of $[0 / \alpha /-\alpha]$}

As for the laminating angle study, a very simple $[0 / \alpha /-\alpha]$ structure is studied at first. While using the $[0 / \alpha /-\alpha]$ pattern as lamination, different choices of the angle $\alpha$ lead to different results.

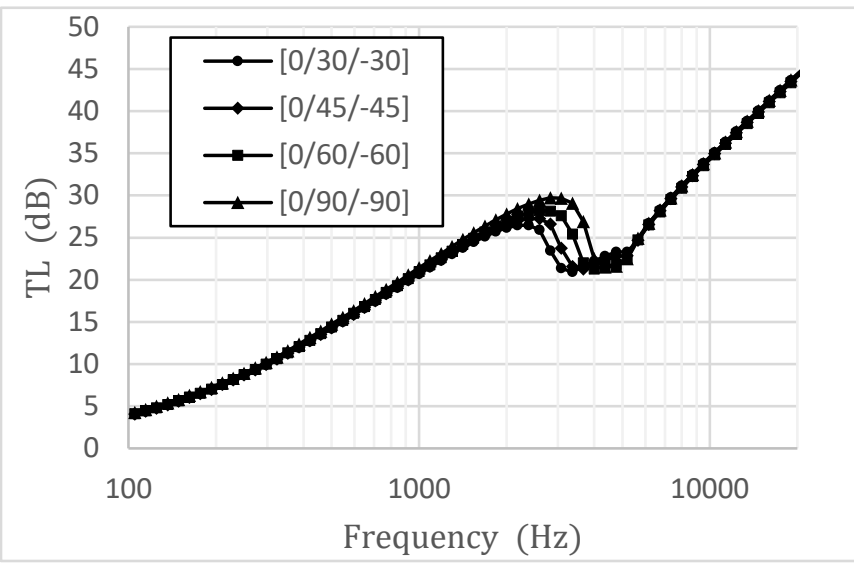

Figure 3 TL comparison of composite patterns with different laminating angles

As shown in the Figure 3, some regularities appear by increasing the laminating angle. The insulation valleys move towards higher frequencies without changing minimum values. Nevertheless, the insulation peaks move towards higher frequencies by keeping rising tendency. Except the band where the insulation valleys appear, there is no distinct difference between different laminating structures. When the angle $\alpha$ equals to 90 degrees, the insulation property seems to be the best in comparison with the others. In this case, the laminated plate is the most isotropy compared to other possible patterns. It can be concluded that, for structures with lamination patterns such as $[0 / \alpha /-\alpha]$, the property of uniform leads to an optimal insulation property. The circumstance shows that the stimulation is characterized by a high singleness on certain frequency, using a specific angle $\alpha$ to avoid the insulation valley may perform better than simply applying a quasi-uniform structure.

\section{Studies on composite patterns in form of $[\boldsymbol{\alpha} / \boldsymbol{\beta} /-\boldsymbol{\beta} /-\boldsymbol{\alpha}]$}

Another structure that is more common in practical engineering is proposed. It is named anti-symmetrical which signify $[\alpha / \beta /-\beta /-\alpha]$ for four-layer example. This time, we would not fix neither $\alpha$ nor $\beta$. For a four-layer laminated antisymmetrical structure, there are in total 42 possibilities by choosing angles from 7 alternatives. Because of the bidirectional identity, only the acute angle is considered in this case. The maximum angle between two consecutive layers is

$$
\max \left\{\begin{array}{c}
(|\beta-\alpha|, 2 \beta) \quad \text { if } \beta \leq 45^{\circ} \\
\left(|\beta-\alpha|, 180^{\circ}-2 \beta\right) \text { if not }
\end{array}\right.
$$

And the minimum angle between two consecutive layers is

$$
\min (|\beta-\alpha|, 2 \beta)
$$

The following table shows the insulation properties of a set of structures where $\alpha$ equals to 0 degree.

\begin{tabular}{|ccc|}
\hline $\begin{array}{c}\text { Laminate } \\
\text { structure }\end{array}$ & $\begin{array}{c}\text { Local maximum } \\
\text { TL }(\mathrm{dB})\end{array}$ & $\begin{array}{c}\text { Occurrence } \\
\text { frequency }(\mathrm{Hz})\end{array}$ \\
\hline$[0 / 15 /-15 / 0]$ & 27.99 & 3084.4 \\
{$[0 / 30 /-30 / 0]$} & 28.03 & 3174.8 \\
{$[0 / 45 /-45 / 0]$} & 28.13 & 3267.8 \\
{$[0 / 60 /-60 / 0]$} & 28.28 & 3267.8 \\
{$[0 / 75 /-75 / 0]$} & 28.44 & 3363.6 \\
{$[0 / 90 /-90 / 0]$} & 28.50 & 3462.2 \\
\hline
\end{tabular}

Table 1 Synthesis of antisymmetric structure simulations' result

Some sound insulation performance law can be summarized in the mid-high frequency band from the numerical experiments. When the total mass is controlled, the local minimum value of sound insulation will not be related to the laminating manners. While the local maximum increases with the increment of angle between two laminates. The frequency where the local minimum occurs increases with the increment of intersection angle as well. It should be pointed out that these laws work only for the classical antisymmetrical laminating plate.

When using some other composite material with the same type of fiber-reinforced for the same numerical experiments, very similar results were produced. Starting from the frequency band in which the low frequency resonance dominates, the vibration of the composite sheet begins to enter the mass dominating zone as explained by the 'mass law'. In 
this area, the sound insulation of the board is affected by the surface mass density and the excitation frequency. If the surface mass density of the laminated plate is the same as that of the single-layer aluminum alloy panel, there is no significant difference between them on sound insulation performance and the sound insulation in the mass control zone. The composite panel and the general material homogenous panel differ in the middle and high frequency. The sound insulation valley of a composite laminate occurs in a frequency domain that is lower than that of a generally homogeneous material, both the laminating angle and sequence will affect the location of the local minimum, as can be seen from the various figures in the precedent paragraphs. When the structure of the laminate remains the same, increasing the angle between the layers as much as possible can shift the frequency of local minimum to a high frequency. In addition, we find that the minimum sound insulation value basically tends to a certain value when the surface density is identical. Therefore, it can be concluded that when controlling the same surface mass density and the thickness of each layer, the maximum interlayer angle can achieve better sound insulation performance in the high frequency band. A structure with large angle between the laminates is equivalent to that with a more macroscopical isotropy in the plane of the plates.

\section{CONCLUSIONS}

Some interesting results are drawn through the theoretical studies and numerical experiments:

1. The 'mass law' dominates the vibro-acoustic performance of composite laminated plates in the low frequency domain.

2. In the middle and high frequency domain, the vibroacoustic performance of composite laminated plates turns out to be more complicated. Both the laminating angle and sequence will affect the sound transmission loss values.

3. Three typical lamination patterns in form of $[0 / \alpha]$, $[0 / \alpha /-\alpha]$ and $[\alpha / \beta /-\beta /-\alpha]$ are studied.

According to the numerical experiments' results, we have concluded that: First, for the $[0 / \alpha]$ pattern, the proportion of these two selected angles do not influence the insulation property; Second, for the $[0 / \alpha /-\alpha]$ pattern, when the structure of the laminate remains the same, increasing the angle between the layers as much as possible can shift the frequency of local minimum to a higher frequency; Third, for the $[\alpha / \beta /-$ $\beta /-\alpha]$ pattern, better sound insulation property can be achieved when the lamination is conducted as uniform as possible.

Although the numerical experiments are carried out on simple models and hypothesis are adopted to simplify the calculations, the sensitivity analysis provides a general guidance to practical application in engineering. Once the application circumstance is determined, using specific laminating structure as shown in this paper helps to avoid acoustic insulation valley and improve structural properties. Due to its excellent physical and chemical properties, composite materials have very good application prospects in many fields, including some possibilities that have not yet been discovered. This study only gives the basic idea of exploring the sound insulation properties of laminates. It will be interesting to apply some special boundary conditions to the composite laminate or to add some special structures such as honeycomb materials between the consecutive plates. Furthermore, a realistic experiment in the standing wave tube may give the results more convincing and useful. We hope that the study can not only give a basic reference to engineering application, but also inspire the researchers who are interested in composite laminates' acoustic properties.

\section{References}

Rayleigh, J. W. S. B. (1878). The theory of sound (Vol. 1). Macmillan

Rayleigh, J. W. S. B. (1896). The theory of sound (Vol. 2). Macmillan

Cremer, L. (1942). Theorie der Schalldämmung dünner Wände bei schrägem Einfall. Akust. Z, 7(3), 81

Shorter, P. J., Cotoni, V., \& Langley, R. S. (2005). Numerical and Experimental Validation of the Hybrid FESEA method

Li S., Zhao D. Y. (2001). Effect of the layup geometry of composite laminates on structural acoustic transmission. Vibration and Shock, 20(2), 86-88

Yan C. X., Huang J. (2016). Numerical Simulation and Experimental Study on Sound Insulation Performance of Sound Insulation Composites. Engineering Plastics Application, 7(7)

Sastry J. S., \& Munjal M. L. (1995). A transfer matrix approach for evaluation of the response of a multi-layer infinite plate to a two-dimensional pressure excitation. Journal of sound and vibration, 182(1), 109-128.

Beranek L. L., \& Mellow T. (2012). Acoustics: sound fields and transducers. Academic Press.

Reixach R., Del Rey R., Alba J., Arbat G., Espinach F. X., \& Mutjé P. (2015). Acoustic properties of agroforestry waste orange pruning fibers reinforced polypropylene composites as an alternative to laminated gypsum boards. Construction and Building Materials, 77, 124-129.

Tsai Y. T., Pawar S. J., \& Huang J. H. (2015). Optimizing material properties of composite plates for sound transmission problem. Journal of Sound and Vibration, 335, 174-186.

Meng G., \& Yan Y. (2017). Vibration and Acoustics of Composite Structures. Beijing: National Defence Industry Press.

Zhang Z., \& Du Y. (2017). Sound insulation analysis and optimization of anti-symmetrical carbon fiber reinforced polymer composite materials. Applied Acoustics, 120, 34-44.

Sheng M., Wang M., \& Sun J. (2001). The basis of noise and vibration control technology. Beijing: Science Press.

Du G., Zhu Z., \& Gong X. (2001). Acoustics Foundation. Nanjing: Nanjing University Press. 